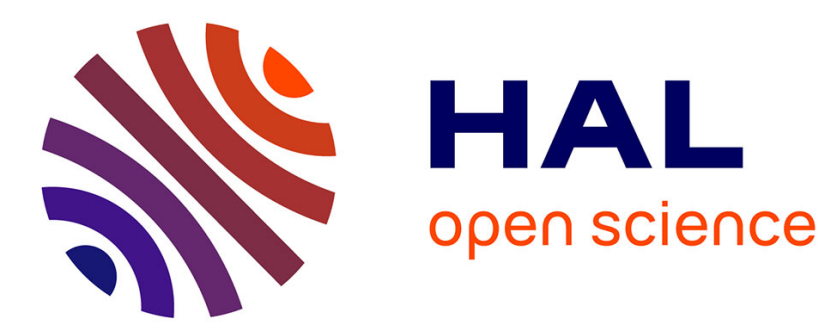

\title{
Three-dimensional imaging of flat natural and cultural heritage objects by a Compton scattering modality
}

\author{
Mai K. Nguyen, Laurent Dumas, Serge X. Cohen, Patricio Guerrero
}

\section{To cite this version:}

Mai K. Nguyen, Laurent Dumas, Serge X. Cohen, Patricio Guerrero. Three-dimensional imaging of flat natural and cultural heritage objects by a Compton scattering modality. Journal of Electronic Imaging, 2017, 26 (1), pp.11026 - 11026. 10.1117/1.JEI.26.1.011026 . hal-01472451

\section{HAL Id: hal-01472451 \\ https://hal.science/hal-01472451}

Submitted on 5 Apr 2018

HAL is a multi-disciplinary open access archive for the deposit and dissemination of scientific research documents, whether they are published or not. The documents may come from teaching and research institutions in France or abroad, or from public or private research centers.
L'archive ouverte pluridisciplinaire HAL, est destinée au dépôt et à la diffusion de documents scientifiques de niveau recherche, publiés ou non, émanant des établissements d'enseignement et de recherche français ou étrangers, des laboratoires publics ou privés. 


\section{Electronnic Imaging}

\section{Three-dimensional imaging of flat natural and cultural heritage objects by a Compton scattering modality}

Patricio Guerrero Prado

Mai K. Nguyen

Laurent Dumas

Serge X. Cohen 


\title{
Three-dimensional imaging of flat natural and cultural heritage objects by a Compton scattering modality
}

\author{
Patricio Guerrero Prado, ${ }^{a, b, c}$ Mai K. Nguyen, ${ }^{b}$ Laurent Dumas, ${ }^{c}$ and Serge X. Cohen ${ }^{a, *}$ \\ aIPANEMA, CNRS/Ministère de la culture et de la communication/Université de Versailles, USR 3461, BP48 Saint-Aubin, \\ 91192 Gif-sur-Yvette, France \\ 'Équipes Traitement de l'Information et Systèmes, ENSEA/Université de Cergy-Pontoise/CNRS UMR 8051, 2 rue Adolphe Chauvin, \\ 95302 Cergy-Pontoise, France \\ 'Laboratoire de Mathématiques de Versailles, CNRS/Université de Versailles UMR 8100, 45 avenue des Etats-Unis, 78035 Versailles, France
}

\begin{abstract}
Characterization and interpretation of flat ancient material objects, such as those found in archaeology, paleoenvironments, paleontology, and cultural heritage, have remained a challenging task to perform by means of conventional x-ray tomography methods due to their anisotropic morphology and flattened geometry. To overcome the limitations of the mentioned methodologies for such samples, an imaging modality based on Compton scattering is proposed in this work. Classical $\mathrm{x}$-ray tomography treats Compton scattering data as noise in the image formation process, while in Compton scattering tomography the conditions are set such that Compton data become the principal image contrasting agent. Under these conditions, we are able, first, to avoid relative rotations between the sample and the imaging setup, and second, to obtain three-dimensional data even when the object is supported by a dense material by exploiting backscattered photons. Mathematically this problem is addressed by means of a conical Radon transform and its inversion. The image formation process and object reconstruction model are presented. The feasibility of this methodology is supported by numerical simulations. (C) The Authors. Published by SPIE under a Creative Commons Attribution 3.0 Unported License. Distribution or reproduction of this work in whole or in part requires full attribution of the original publication, including its DOI. [DOI: 10.1117/1.JEI .26.1.011026]
\end{abstract}

Keywords: Radon transform; Compton scattering tomography; ancient material objects; image reconstruction.

Paper 16582SS received Jul. 1, 2016; accepted for publication Nov. 29, 2016; published online Feb. 8, 2017.

\section{Introduction}

\subsection{Context}

Material characterization of ancient flat object encountered in natural or cultural heritage studies remains notably challenging with nowadays $x$-ray imaging methods. When one deals with heritage objects, the noninvasiveness and nondestructiveness properties of inspections are a requirement that $\mathrm{x}$-ray imaging methodologies provide, enabling two-dimensional (2-D) imaging of the sample. However, one can easily be facing samples presenting a flattened geometry, i.e., samples presenting a large ratio between its front area and thickness. The challenge is then to perform a three-dimensional (3-D) probing without using a relative rotation between the sample and the imaging setup as would be done in conventional tomography, using either absorption or phase contrast modality, since probing would suffer from the high differential light path in distinct directions.

Samples presenting such characteristics are encountered in, e.g., studies of conservation/restoration of easel paintings requiring the characterization of the stratigraphical assemblage of pigments often over a very dense background layer such as one made of lead white. An example of this kind of study is shown in Fig. 1 which was performed by means of an invasive method. Figure 2 presents another example of objects possessing this morphology, namely in paleontology with the Lagerstätten fossils ${ }^{1}$ which are mechanically flattened during the fossilization process and

*Address all correspondence to: Serge X. Cohen, E-mail: serge.cohen@ synchrotron-soleil.fr stand on one side of a thick sedimental slab which cannot be thinned for the study. In both cases, the volume of interest forms a layer on top of a material support which is opaque to $\mathrm{x}$-rays either due to its density or its thickness.

Two alternatives are currently available to work out this issue : either to perform a stratigraphical section of the sample which is an invasive method, or to limit the study to a bidimensional analysis limited to the front surface of the sample, for example with synchrotron x-ray fluorescence spectral raster-scanning as performed in Ref. 1, none of which enabling a 3-D study of the sample. Furthermore, because of the opaque supporting material, transmission, and forward scattering data, i.e., with a scattering angle inferior to $\pi / 2$, is impossible to collect. This motivates the proposal of a modality using backscattered data, i.e., data collected with a scattering angle $\omega$ comprised between $\pi / 2$ and $\pi$, as shown in Fig. 3. In the following, we will call $\bar{\omega}=\pi-\omega$ the supplementary angle of $\omega$.

Identity (1) introduces the Compton equation, a diffeomorphism between the scattering angle and the scattered energy under the hypotheses that electrons are both free and at rest and that the incident beam is monochromatic, i.e., a single value $E_{0}$ of an incident energy irradiating the object. Equation (1) provides a reliable approximation of a real scattering scenario and with it, the scattering image formation process corresponds to a Radon transform over the surface of a cone with a fixed axis direction. ${ }^{2-4}$

\subsection{Compton Scattering Tomography}

For the energy range from $50 \mathrm{eV}$ up to $1 \mathrm{MeV}$, the dominant photon-matter interaction processes are the photoelectric 


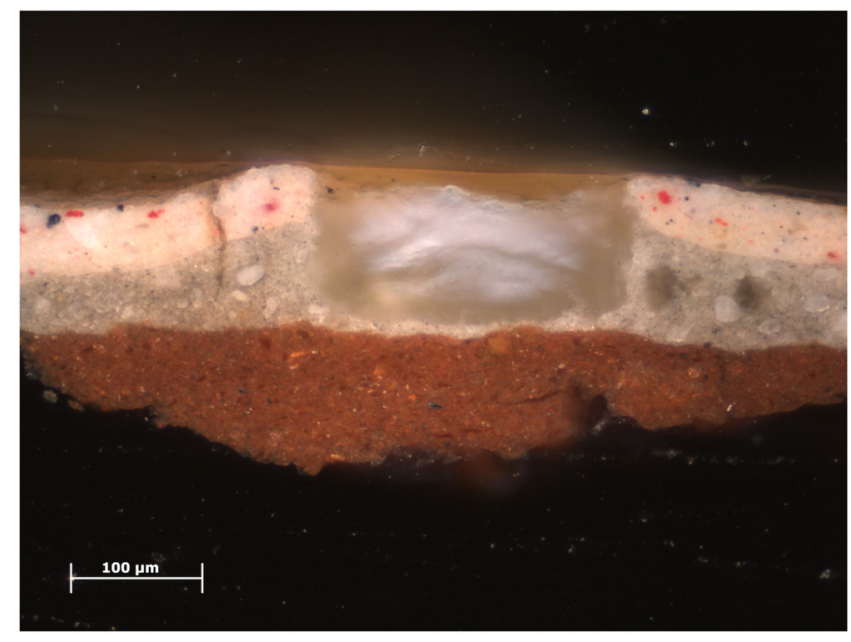

Fig. 1 Paint cross-section showing a stratigraphical assemblage of The Anatomy Lesson of Dr. Nicolaes Tulp, 1632 by Rembrandt, Mauritshuis, The Hague. (C) Sample taken and prepared as cross-section by $P$. Noble during the conservation treatment of the painting in 1997 and rephotographed by A. van Loon, Mauritshuis, in 2010 for the Rembrandt Database.

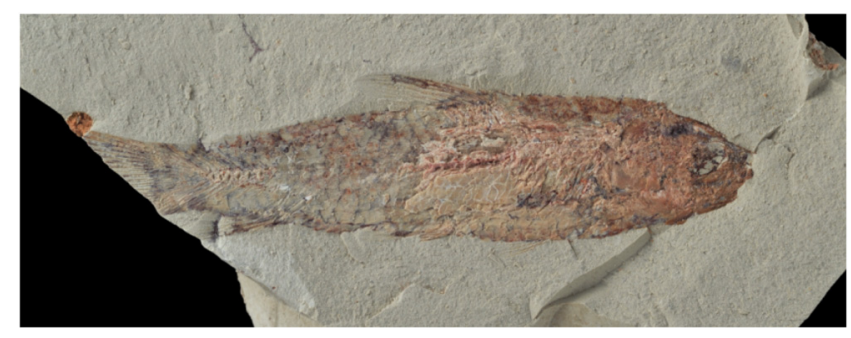

Fig. 2 A flat fossil actinopterygian over a thick, highly x-ray absorbing support from the Kem Kem Beds in Morocco dated back to the Lower Cretaceous (95 million years ago). () P. Gueriau (MHNM/MNHN).

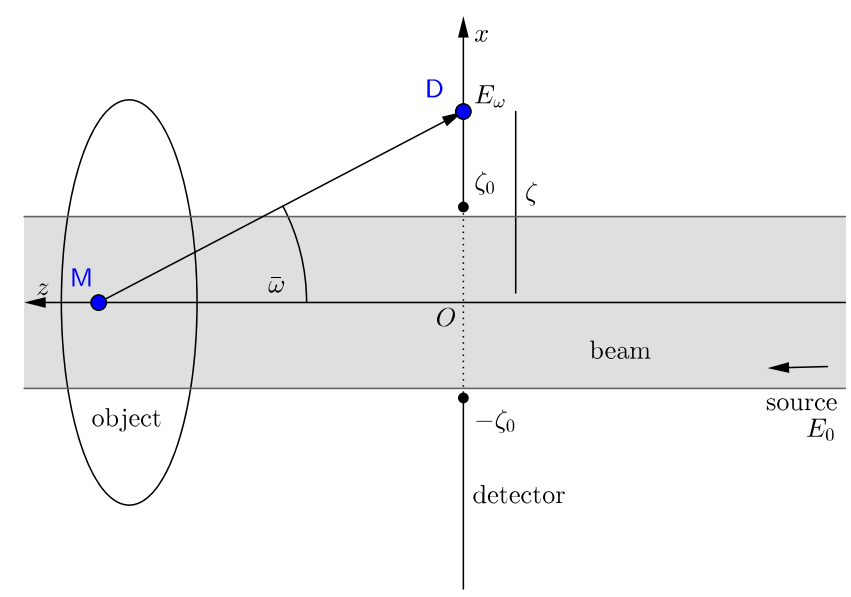

Fig. 3 Compton backscattering fundamentals. A photon of energy $E_{0}$ is Compton scattered by an angle $\omega=\pi-\bar{\omega}$ generating a new photon of energy $E_{\omega}$.

absorption, Rayleigh scattering which is both elastic and coherent, and Compton scattering which conversely is both inelastic and incoherent. In this last one, an incident photon of energy $E_{0}$ is absorbed by a target electron, which re-emits a secondary photon scattered by an angle $\omega$ relative to the direction of the original photon. The scattered photon has then an energy $E_{\omega}$ which is related to the scattering angle $\omega$ by Eq. (1), the Compton equation, presented later.

In classical x-ray imaging and tomography, the Compton scattered signal is considered as noise added to photoelectric absorption and coherent scattered data. This is because the $\mathrm{X}$-ray transmission signal is dominated by the photoelectric absorption while coherent scattering may produce significant amplitude variations at low scattering angles because of constructive and destructive interference effects due to the coherent nature of this scattering. However, depending on the material, if the incident radiation has a superior energy of about $4 \times 10^{4}$ electron-volts, Compton scattering becomes the dominant phenomenon in the process, even more when detection is performed outside the direct transmission area $(\omega=0)$.

Classical tomographic imaging modalities developed and used in most all applications in the last half century include transmission computed tomography, single photon emission tomography, and positron emission tomography. All of them regard primary radiation and perform 3-D mappings leaning on relative rotations between the object and the imaging setup. In such a framework, Compton scattering events are adding a nonuniform background to the observation, a systematic bias which leads to artifacts in the reconstruction if it is not accounted for. As the relative importance of the Compton scattering effect over the other two processes mentioned above is increasing with an increase on incident energy, its effect is even more important when using higher energy $\gamma$-ray imaging.

The idea of exploiting scattered radiation from the Compton effect in imaging techniques has been introduced and studied simultaneously and it has given birth to CST, ${ }^{2,4-8}$ which focuses on reconstructing the electron density map of the object.

To compensate the information provided by multiple projection angles in classical tomography, CST exploits the energy loss of the scattered photons. This energy loss is related to the scattering angle via the Compton equation given by

$E_{\omega}=\frac{E_{0}}{1+\frac{E_{0}}{m_{e} c^{2}}(1-\cos \omega)}$,

where $m_{e} c^{2}=511 \mathrm{keV}$ is the rest mass energy of the electron.

The first CST scanner was proposed in $1994^{5}$ through a Radon transform over an arc of circles starting at a $\gamma$-ray source and ending at a fixed detecting site. Radon transforms over conical surfaces having fixed axis directions and variable opening angle are studied in Ref. 2 where a first analytic inversion equation is proposed using circular component analysis, with applications to emission imaging based on Compton scattered radiation. Generalizations of this kind of transform to higher dimensions spaces with related inverse formulas in a filtered backprojection type are presented in Ref. 3. A backprojection inversion algorithm for a conical Radon transform in $\mathbb{R}^{3}$ was recently developed in Ref. 4.

This paper is organized as follows. Section 2 describes the imaging configuration. Section 3 defines the conical Radon transform and presents the image formation process. 


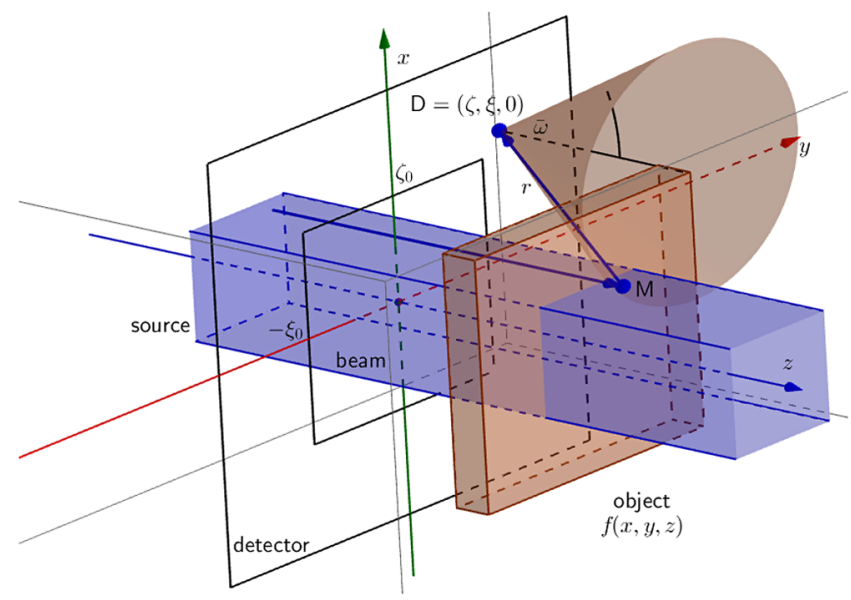

Fig. 4 Imaging configuration proposed. A scattering site $M$ produces scattered radiation captured at a detecting site $D$. The incident photon is coming from a parallel beam with a square cross-section.

Section 4 details object reconstructions via a backprojection inversion from Ref. 4. In Sec. 5, we present a numerical scheme and simulation results for flat objects both for energy resolved image formation and sample reconstruction. Finally, Sec. 6 closes this work with conclusions and perspectives.

\section{Proposed Setup}

CST aims to reconstruct the electron density map of the studied object. In this work, the electron density is represented mathematically by a nonnegative bump function (both smooth and of bounded support) $f: \mathbb{R}^{2} \times \mathbb{R}^{+} \rightarrow \mathbb{R}^{+}$.

Essentially, as shown in Fig. 4, an incident photon of energy $E_{0}$, belonging to a monochromatic parallel beam with a square section, is Compton scattered by an electron situated inside the object at $M$ subtending an angle $\omega$ $\left(\frac{\pi}{2}<\omega<\pi\right)$ with the direction of incidence. The scattered photon, of energy $E_{\omega}$ approximated by Eq. (1), reaches a detecting site $\mathrm{D}=(\zeta, \xi, 0)$ on the 2-D detector that is located over the $x O y$-plane. The parallel beam is centered at the $O z$-axis.

The imaging configuration is described then as follows: as mentioned, a synchrotron radiation setup with a parallel monochromatic x-ray beam (about $50 \mathrm{keV}$ ) and a spaceenergy resolved detector are considered. As represented in Fig. 4, the detector will be placed between the source and the object to capture backscattered photons. It will have a hole in the middle of area $4 \zeta_{0} \xi_{0}$ for some two positive real numbers $\left(\zeta_{0}, \xi_{0}\right)$ to allow the beam to go through. Therefore, we will have data for values of $(\zeta, \xi)$ on the $x O y$-plane verifying $|\zeta|>\zeta_{0}$ or $|\xi|>\xi_{0}$. Horizontal and vertical translations of the sample will be needed to allow imaging of the full object.

\section{Image Formation}

\subsection{Conical Radon Transform}

The conical Radon transform ${ }^{2,3}$ integrates a function over circular cone surfaces; its backprojection reconstruction procedure is developed in Ref. 4.

A $(\psi, r)$-parametrization of the lateral surface of a circular cone is considered, where $\varphi$ is the azimuthal angle and $r$ is the distance of a point in the cone surface from its vertex. Such parametrization reads, for a cone with vertex at $\mathrm{D}=(\zeta, \xi, 0)$ and opening angle $\omega$, noted $\mathcal{C}_{\omega, \mathrm{D}}$

$\mathcal{C}_{\omega, D}=(\zeta+r \sin \omega \cos \psi, \xi+r \sin \omega \sin \psi, r \cos \omega)$.

Therefore, the CRT applied on an electron density function $f$, noted $C f$, defined as the integral of this function over the surface of a cone parametrized as Eq. (2) reads

$$
\begin{aligned}
C f(\zeta, \xi, \omega)= & \int_{0}^{\infty} \int_{0}^{2 \pi} \frac{1}{r} f(\zeta+r \sin \omega \cos \psi, \xi \\
& +r \sin \omega \sin \psi, r \cos \omega) \mathrm{d} \psi \mathrm{d} r .
\end{aligned}
$$

The factor $1 / r$ comes from the integration measure on the cone $r \sin \omega \mathrm{d} \psi \mathrm{d} r$ and the approximation of the solid angle $\frac{1}{r^{2}} \cos \omega$, the factor $\sin \omega \cos \omega$ not appearing in the last integral will be taken into account later in the scattered photon flux time density in Eq. (9).

It may be useful to express Eq. (3) as an integral with respect to $z=r \cos \omega$ and introducing the variable $t=\tan \omega$. If notation $C f$ is conserved, Eq. (3) is also expressed as

$$
\begin{aligned}
C f(\zeta, \xi, t)= & \int_{0}^{\infty} \int_{0}^{2 \pi} \frac{1}{z} f(\zeta+t z \cos \psi, \xi \\
& +t z \sin \psi, z) \mathrm{d} \psi \mathrm{d} z
\end{aligned}
$$

\subsection{Scattered Photon Flux Time Density}

Let $I\left(E_{\omega}, \mathrm{D}\right)$ represent the recorded scattered photon flux density (number of photons of energy $E_{\omega}$ recorded per unit time at D). It incorporates the following parameters:

- $I_{0}$ : the incident photon flux time density just before the scattering event at $\mathrm{M}$.

- $\sigma(\omega)$ : the Klein-Nishina differential cross-section ${ }^{9}$ at an angle $\omega$.

- $f(\mathrm{M})$ : the electron density at M.

- $\mathrm{d} \Omega(\mathrm{M}, \mathrm{D})$ : the solid angle from $M$ to $D$.

- $\mathrm{dM}$ : the area element around M over the cone surface.

The solid angle $d \Omega(M, D)$ can be seen from Fig. 4 to be

$\mathrm{d} \Omega(\mathrm{M}, \mathrm{D})=4 \arctan \left(\frac{1}{4 r^{2}} \tau \cos \bar{\omega}\right)$,

where $\tau$ is the area of the detecting element located at $\mathrm{D}$ and $r$ is the Euclidean distance from $M$ to $D$.

If $\tau$ is small enough, then $\mathrm{d} \Omega(\mathrm{M}, \mathrm{D})$ can be approximated by $\frac{1}{r^{2}} \tau \cos \bar{\omega}$.

The Klein-Nishina differential cross-section is a function of the scattering angle $\omega$ giving the probability of a photon to be scattered in a given direction $\omega$ when the azimuthal angle is uniformly distributed in the interval $(0,2 \pi)$. It is given by ${ }^{9}$

$\sigma(\omega)=\frac{1}{2} r_{e}^{2}\left(\frac{E_{\omega}}{E_{0}}\right)^{2}\left(\frac{E_{\omega}}{E_{0}}+\frac{E_{0}}{E_{\omega}}-\sin ^{2} \omega\right)$, 
where $r_{e}$ is the classical electron radius. The factor $\frac{1}{2} r_{e}^{2}$ comes from the diametrical transversal area of an electron $\pi r_{e}^{2}$ and the uniformity of the azimuthal angle $\frac{1}{2 \pi}$.

Consequently, the scattered photon flux density at D, given a scattering site $M$, reads

$\mathrm{d} I\left(E_{\omega}, \mathrm{D} \mid \mathrm{M}\right)=I_{0} \sigma(\omega) \mathrm{d} \Omega(\mathrm{M}, \mathrm{D}) f(\mathrm{M}) \mathrm{dM}$.

The total scattered flux time density $I\left(E_{\omega}, \mathrm{D}\right)$ recorded at $\mathrm{D}$ is the integral over all scattering sites laying over the surface of the cone $\mathcal{C}_{\bar{\omega}, \mathrm{D}}$. It is hence given by the integral

$I\left(E_{\omega}, \mathrm{D}\right)=\int_{M \in \mathcal{C}_{\bar{\omega}, \mathrm{D}}} \mathrm{d} I\left(E_{\omega}, \mathrm{D} \mid \mathrm{M}\right)$.

From the last integral, we can extract the conical Radon transform, and we are able to express the scattered photon flux density for a recorded energy $E$ as

$I(E, \mathrm{D})=\tau I_{0} \sin \bar{\omega}_{E} \cos \bar{\omega}_{E} \sigma\left(\omega_{E}\right) C f\left(\bar{\omega}_{E}, \zeta, \xi\right)$.

Note the use of $\omega_{E}$ and $\bar{\omega}_{E}$, the scattering angle and its supplementary, related to the recorded energy $E$ approximated by an inversion of identity (1).

\section{Object Reconstruction}

\subsection{Bessel Function and Hankel Transform}

We recall the Bessel function of the first kind of order 0 , and the Hankel transform of a function $f$ of order 0 noted, respectively, $J_{0}$ and $h_{0}$,

$J_{0}(x)=\frac{1}{2 \pi} \int_{0}^{2 \pi} e^{i x \sin \alpha} \mathrm{d} \alpha$

$h_{0} f(k)=\int_{0}^{\infty} f(r) J_{0}(k r) r \mathrm{~d} r$

The inverse expression of the Hankel transform reads

$f(r)=\int_{0}^{\infty} h_{0} f(k) J_{0}(k r) k \mathrm{~d} k$

\subsection{Inverse Formula}

We start writing the bidimensional $(\zeta, \xi)$-Fourier transform of Eq. (4) as

$$
\begin{aligned}
\widehat{C f}(u, v, t)= & \int_{0}^{\infty} \int_{0}^{2 \pi} \int_{\mathbb{R}^{2}} \frac{1}{z} f(\zeta+t z \cos \psi, \xi \\
& +t z \sin \psi, z) e^{-2 \pi i(u \zeta+v \xi)} \mathrm{d} \zeta \mathrm{d} \xi \mathrm{d} \psi \mathrm{d} z
\end{aligned}
$$

and with a change of variables (translation of the vertex to the origin) $x=\zeta+t z \cos \psi$ and $y=\xi+t z \sin \psi$, Eq. (13) becomes

$$
\begin{aligned}
\widehat{C f}(u, v, t)= & \int_{0}^{\infty} \int_{0}^{2 \pi} \frac{1}{z} \int_{\mathbb{R}^{2}} f(x, y, z) e^{-2 \pi i(u x+v y)} \mathrm{d} x \mathrm{~d} y \\
& \times e^{2 \pi i t z(u \cos \psi+v \sin \psi)} \mathrm{d} \psi \mathrm{d} z,
\end{aligned}
$$

where we recognize the $(x, y)$-Fourier transform of $f$, then the last expression reads

$$
\widehat{C f}(u, v, t)=\int_{0}^{\infty} \frac{1}{z} \hat{f}(u, v, z) \int_{0}^{2 \pi} e^{2 \pi i t z(u \cos \psi+v \sin \psi)} \mathrm{d} \psi \mathrm{d} z
$$

where we can identify the Bessel function of the first kind of order 0 by switching to polar coordinates via $u=q \cos \beta$ and $v=q \sin \beta$ by

$$
\begin{aligned}
\widehat{C f_{p}}(q, \beta, t) & =\int_{0}^{\infty} \frac{1}{z} \hat{f}_{p}(q, \beta, z) \int_{0}^{2 \pi} e^{2 \pi i q t z \cos (\beta-\psi)} \mathrm{d} \psi \mathrm{d} z \\
& =2 \pi \int_{0}^{\infty} \frac{1}{z} \hat{f}_{p}(q, \beta, z) J_{0}(2 \pi q t z) \mathrm{d} z
\end{aligned}
$$

Note the use of notation $\widehat{C f_{p}}$ and $\hat{f}_{p}$ to point out the use of polar coordinates.

The last integral turns out to be the Hankel transform of the function $g: z \rightarrow \frac{1}{z^{2}} \hat{f}_{p}(q, \beta, z)$, then Eq. (16) reads

$\widehat{C f_{p}}(q, \beta, t)=2 \pi h_{0} g(2 \pi q t)$.

One can, therefore, apply the Hankel inverse equation to have

$g(z)=\frac{1}{2 \pi} \int_{0}^{\infty} \widehat{C f_{p}}(q, \beta, t) J_{0}(2 \pi q z t) 2 \pi q t \mathrm{~d}(2 \pi q t)$.

Then going back to $\hat{f}_{p}$ from $g$, we have

$\hat{f}_{p}(q, \beta, z)=2 \pi z^{2} q^{2} \int_{0}^{\infty} \widehat{C f_{p}}(q, \beta, t) J_{0}(2 \pi q z t) \mathrm{d} t$.

Finally take the inverse Fourier transform in polar coordinates to recover $f$ as

$$
\begin{aligned}
f(x, y, z)= & 2 \pi z^{2} \int_{0}^{2 \pi} \int_{0}^{\infty} \int_{0}^{\infty} \widehat{C f_{p}}(q, \beta, t) J_{0}(2 \pi q t z) t \mathrm{~d} t q^{3} \\
& \times e^{2 \pi i q(x \cos \beta+y \sin \beta)} \mathrm{d} q \mathrm{~d} \beta .
\end{aligned}
$$

The last integral may be expressed in Cartesian coordinates related to the Fourier domain $(u, v)$ and in terms of $\omega$, then we are able to write the inverse equation of Eq. (3) as

$$
\begin{aligned}
& f(x, y, z)=2 \pi z^{2} \int_{\mathbb{R}^{2}}\left(u^{2}+v^{2}\right) e^{2 \pi i(u x+v y)} \\
& \quad \times \int_{0}^{\pi / 2} \frac{\sin \omega}{\cos ^{3} \omega} \widehat{C f}(u, v, \omega) J_{0}\left(2 \pi z \tan \omega \sqrt{u^{2}+v^{2}}\right) \mathrm{d} \omega \mathrm{d} u \mathrm{~d} v .
\end{aligned}
$$

\subsection{Adjoint Transform}

Let us introduce some basic notations. $C$ is defined as an operator from $X:=L_{2}\left(\mathbb{R}^{2} \times \mathbb{R}^{+}\right)$to $Y:=L_{2}\left(\mathbb{R}^{2} \times\left[0, \frac{\pi}{2}\right]\right)$, and the respective inner products of those spaces are defined by

$$
\begin{aligned}
\left(f_{1}, f_{2}\right)_{X} & =\int_{\mathbb{R}} \int_{\mathbb{R}^{2}} f_{1}(x, y, z) f_{2}(x, y, z) \mathrm{d} x \mathrm{~d} y \mathrm{~d} z \\
\left(g_{1}, g_{2}\right)_{Y} & =\int_{\mathbb{R}^{2}} \int_{0}^{\pi / 2} g_{1}(\zeta, \xi, \omega) g_{2}(\zeta, \xi, \omega) \mathrm{d} \omega \mathrm{d} \zeta \mathrm{d} \xi .
\end{aligned}
$$

The adjoint transform $C^{\dagger}$ of $C$, closely related to the backprojection operator, is defined as the transform from $Y$ to $X$ that verifies 
$(g, C f)_{Y}=\left(C^{\dagger} g, f\right)_{X}$.

In order to derive an expression for $C^{\dagger}$, we start from the left side of the last identity and introduce the definition of $C$ given in Eq. (3) writing

$$
\begin{aligned}
(g, C f)_{Y} & =\int_{\mathbb{R}^{2}} \int_{0}^{\pi / 2} g(\zeta, \xi, \omega) C f(\zeta, \xi, \omega) \mathrm{d} \omega \mathrm{d} \zeta \mathrm{d} \xi \\
& =\int_{\mathbb{R}^{2}} \int_{0}^{\pi / 2} g(\zeta, \xi, \omega) \\
& =\int_{0}^{\infty} \int_{0}^{2 \pi} \frac{1}{r} f(\zeta+r \sin \omega \cos \psi, \xi \\
& +r \sin \omega \sin \psi, r \cos \omega) \mathrm{d} \psi \mathrm{d} r \mathrm{~d} \omega \mathrm{d} \zeta \mathrm{d} \xi,
\end{aligned}
$$

and apply a change of variables in the form $x=\zeta+t z \cos \psi$, $y=\xi+t z \sin \psi$, and Fubini's theorem to have

$$
\begin{aligned}
(g, C f)_{Y} & =\int_{\mathbb{R}} \int_{\mathbb{R}^{2}} \frac{1}{z} f(x, y, z) \int_{0}^{\pi / 2} \int_{0}^{2 \pi} g(x-z \tan \omega \cos \psi, y \\
& -z \tan \omega \sin \psi, \omega) \mathrm{d} \psi \mathrm{d} \omega \mathrm{d} x \mathrm{~d} y \mathrm{~d} z .
\end{aligned}
$$

From which one is able to extract the adjoint transform $C^{\dagger}$ of $C$ from identity (23) in the form

$$
\begin{aligned}
C^{\dagger} g(x, y, z)= & \frac{1}{z} \int_{0}^{\pi / 2} \int_{0}^{2 \pi} g(x-z \tan \omega \cos \psi, y \\
& -z \tan \omega \sin \psi, \omega) \mathrm{d} \psi \mathrm{d} \omega .
\end{aligned}
$$

The way it is defined, the adjoint transform can be interpreted here as a backprojection procedure of projections $g(x-z \tan \omega \cos \psi, y-z \tan \omega \sin \psi, \omega)$ to the position $(x, y, z)$ times the factor $1 / z$, i.e., one assigns to $(x, y, z)$ the values of projections starting at this point, forming a cone toward the detector with opening angle $\omega$ times the mentioned factor.

For a first rough reconstruction, $f(x, y, z)$ can be approximated through the adjoint transform or equivalently in this case, through a simple backprojection of data as

$f(x, y, z) \approx C^{\dagger} C f(x, y, z)$.

\subsection{From the Adjoint Transform to Filtered Backprojections}

To show that the adjoint transform, $C^{\dagger}$ is not the inverse operator of $C$, one only needs to compute $C^{\dagger} C f(x, y, z)$ and compare the result with the inversion equation of $C$ Eq. (21) given in Sec. 4.2. Then filters must be added to projections in order to adjust the result of $C^{\dagger} C f(x, y, z)$ in accordance to such an inverse equation.

Therefore, to compute $C^{\dagger} C f(x, y, z)$ from Eq. (23), we insert the bidimensional $(\zeta, \xi)$-Fourier transform of $C$ to have

$$
\begin{aligned}
C^{\dagger} C f(x, y, z) & =\frac{1}{z} \int_{0}^{\pi / 2} \int_{\mathbb{R}^{2}} \widehat{C f}(u, v, \omega) e^{2 \pi i(u x+v y)} \\
& \times \int_{0}^{2 \pi} e^{-2 \pi i z \tan \omega(u \cos \psi+v \sin \psi)} \mathrm{d} \psi \mathrm{d} u \mathrm{~d} v \mathrm{~d} \omega .
\end{aligned}
$$

Last inner $\psi$-integration can be performed via polar coordinates as in Eqs. (15) and (16) allowing us to write the
Bessel function of the first kind of order 0 as

$$
\begin{aligned}
& C^{\dagger} C f(x, y, z)=\frac{2 \pi}{z} \int_{\mathbb{R}^{2}} e^{2 \pi i(u x+v y)} \\
& \quad \times \int_{0}^{\pi / 2} \widehat{C f}(u, v, \omega) J_{0}\left(2 \pi z \tan \omega \sqrt{u^{2}+v^{2}}\right) \mathrm{d} \omega \mathrm{d} u \mathrm{~d} v .
\end{aligned}
$$

The last expression differs from inverse equation (21) only by factors $z^{3}\left(u^{2}+v^{2}\right) \frac{\sin \omega}{\cos ^{3} \omega}$. These factors are seen as filters that we need to apply onto the data to have an exact backprojection inversion procedure.

Therefore, let us define the projections $C^{*} f(\zeta, \xi, \omega)$ filtered in the Fourier space by the filters

$\widehat{C^{*} f}(u, v, \omega)=\left(u^{2}+v^{2}\right) \frac{\sin \omega}{\cos ^{3} \omega} \widehat{C f}(u, v, \omega)$,

and, finally, we are able to write the inverse equation of the CRT in the form

$f(x, y, z)=z^{3} C^{\dagger} C^{*} f(x, y, z)$.

\section{Simulation Results}

A numerical object corresponding to a flattened stratigraphic sample is created, reconstructed by means of inversion equation (31), and presented in Figs. 5-8 for different cross sections of a phantom detailed as follows. The phantom is built with three layers of $512 \times 512 \mu \mathrm{m}^{2}$ regarding its frontal area and about $40 \mu \mathrm{m}$ of thickness per layer, with electron densities of $0.9,1.1$, and 1.0, respectively. Some grains of random diameters and positions are inserted inside the layers, having densities of $1.3 \pm 0.1,6.0 \pm 0.5$, and $2.0 \pm 0.3$ for each layer, respectively.

\subsection{Discretization Parameters}

The unit length considered in discretizations is $4 \mu \mathrm{m}$ thus, each pixel in the images represents $16 \mu \mathrm{m}^{2}$.

A square parallel x-ray beam of $256 \mu \mathrm{m}^{2}$ of sectional area crosses the hole in the detector of $576 \mu \mathrm{m}^{2}\left(\zeta_{0}=\xi_{0}=12 \mu \mathrm{m}\right)$. The medium has dimensions of $512 \times 512 \times 128 \mu \mathrm{m}^{3}$ thus we need 32 translations following the $0 x$-axis and the same for the $O y$-axis, to cover the full medium. The detector plane, located over the $x O y$-plane at a perpendicular distance of $4 \mu \mathrm{m}$ to the sample, has an area of $256 \times 256 \mu \mathrm{m}^{2}$ including the hole. Regarding the number of detecting sites, having an area of 1 pixel each, and considering the absence of detecting sites in the hole, we have 4060 detecting sites.

\subsection{Numerical Image Formation}

Numerical resolution of integral (3) is performed via the trapezoidal rule with a discretization spatial step $\mathrm{d} r=4 \mu \mathrm{m}$ and an azimuthal angular step $\mathrm{d} \psi=0.01 \mathrm{rad}$. 64 opening angles of the cone from 0 to $\pi / 2$ are considered.

\subsection{Numerical Reconstruction}

To numerically compute inversion Eq. (31) from data $C f(\zeta, \xi, \omega)$ generated by Eq. (3), the procedure works as follows:

1. Filter the projections in the Fourier domain to get $\widehat{C^{*} f}(\zeta, \xi, \omega)$ applying Eq. (30).

2. Perform an inverse Fourier transform to obtain the filtered projections $C^{*} f(\zeta, \xi, \omega)$. 
(a)

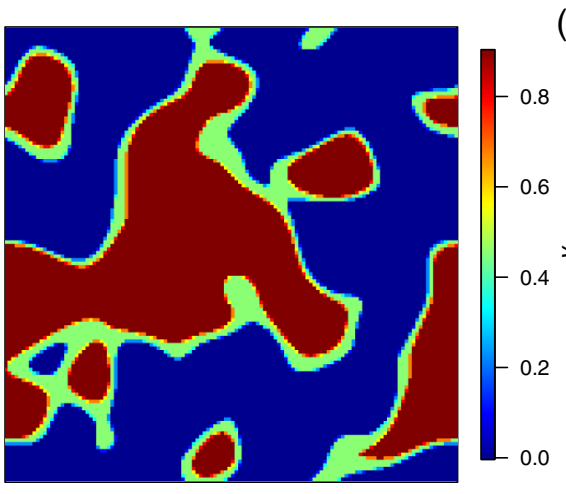

$x$

(c)

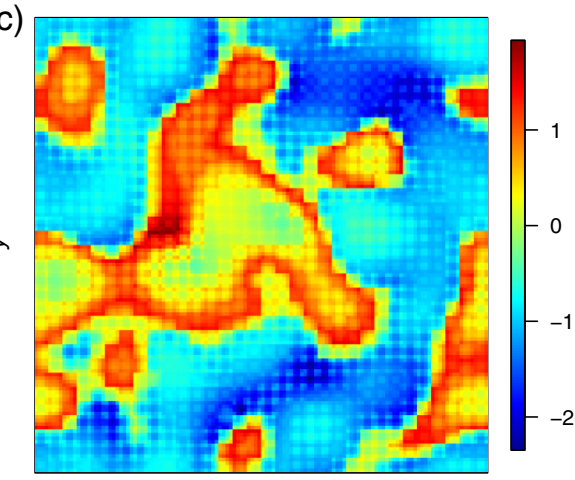

(b)

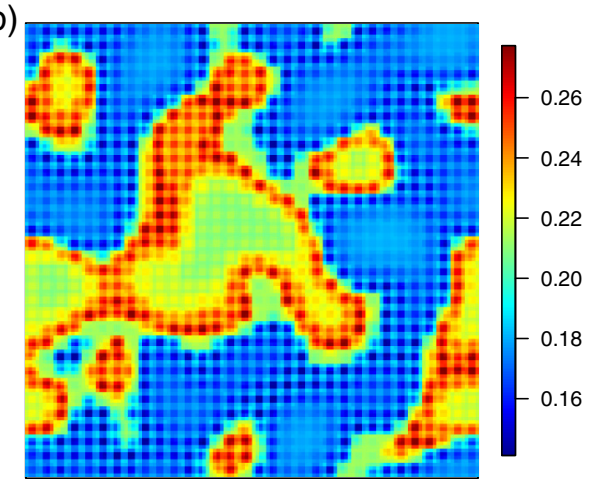

$x$

(d)

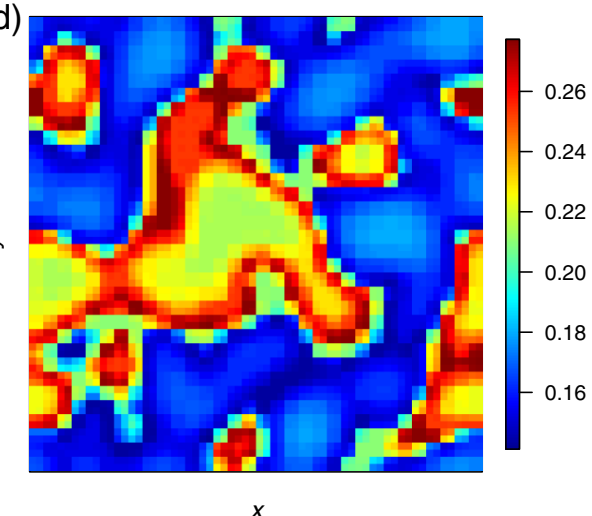

Fig. 5 Stratigraphical phantom of dimensions $128 \times 128 \times 32$ voxels. Plane presented: $z=4$. (a) original phantom, (b) direct inversion, (c) inversion after normalizing values following $z$-axis, and (d) inversion after removing voxels located at edges of single reconstructions of dimensions $4 \times 4 \times 32$ voxels.

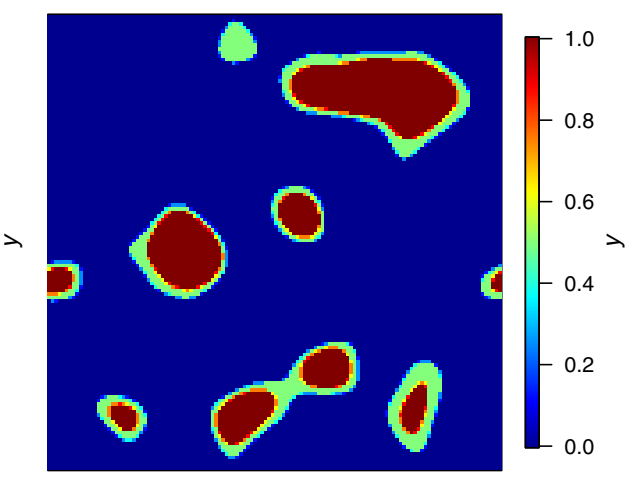

$x$

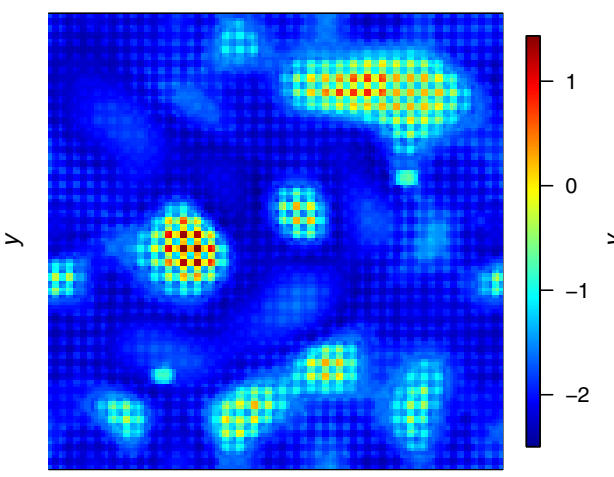

$x$

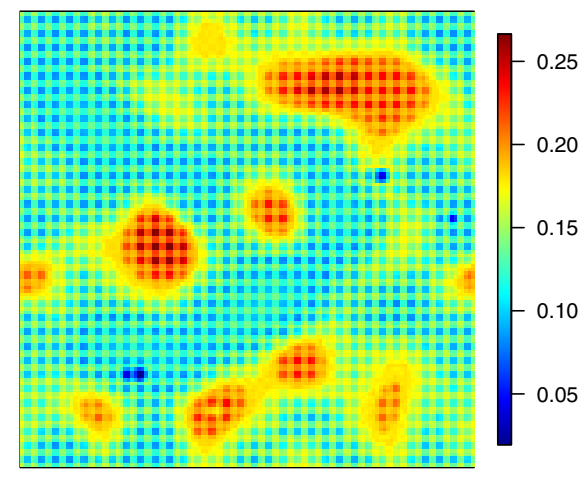

$x$

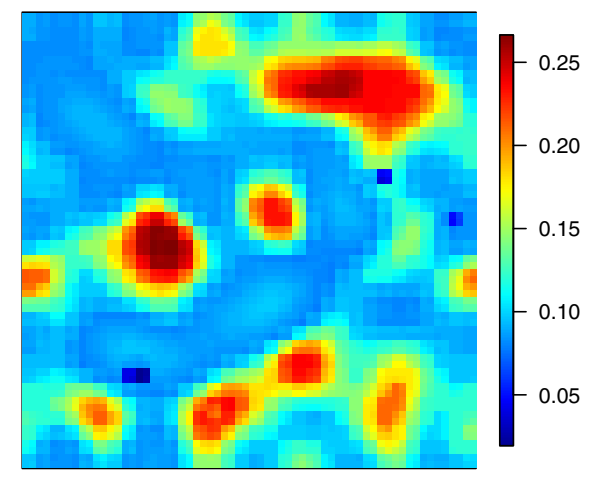

$x$

Fig. 6 Stratigraphical phantom of dimensions $128 \times 128 \times 32$ voxels. Plane presented: $z=30$. Same layout as in Fig. 5. 


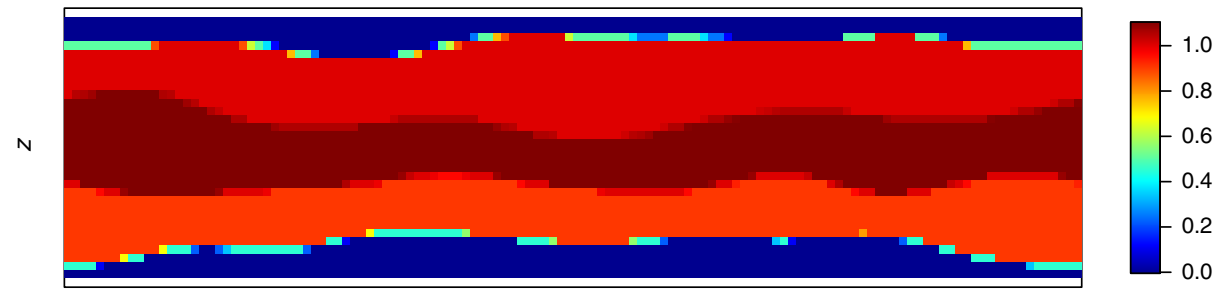

$x$
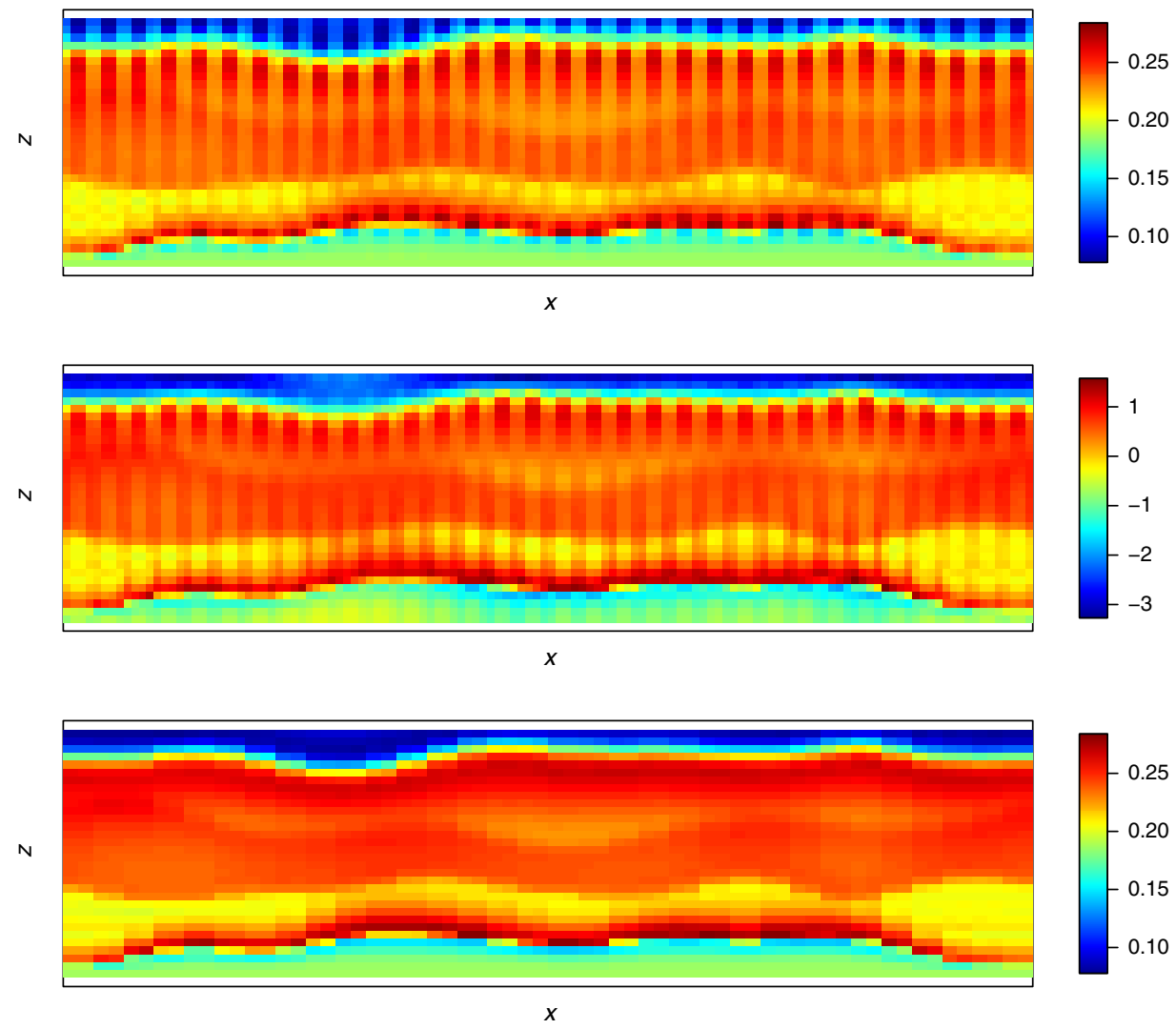

Fig. 7 Stratigraphical phantom of dimensions $128 \times 128 \times 32$ voxels. Plane presented: $y=22$. From top to bottom: original phantom, direct inversion, inversion after normalizing values following $z$-axis, inversion after removing voxels located at edges of single reconstructions of dimensions $4 \times 4 \times 32$ voxels.

3. The backprojection procedure is performed by means of the adjoint transform $C^{\dagger}$ by Eq. (26).

4. Finally, applying the factor $z^{3}$, we compute the inversion Eq. (31).

\subsection{Inversion Procedure Details}

Direct application of Eq. (31) is first presented in figures, just after the original sample, and one can see edge artifacts due to horizontal and vertical translations of the sample and the different scale rates between voxels located at the edge of a single reconstruction and those located at the center. First, the obvious solution presented is to normalize scales following the $z$-axis direction, where we can still see these edge artifacts, and conclude that problem is not only about scaling. In the last solution presented, we removed these voxels located at edges, where we can appreciate an encouraging 3$\mathrm{D}$ reconstruction of the sample.

A cosine window function was used in the Fourier domain of projections to control high frequencies. Data not recorded in the hole area were filled by a numerical solution of a heat diffusion problem from values of energies captured at the hole edge.

\section{Conclusion and Perspectives}

An x-ray imaging modality based on Compton scattering is presented; the interest is to perform a 3-D mapping of flat heritage objects without relying on relative rotations among object, source, and detectors. Both image formation by means of a conical Radon transform and object reconstruction by a filtered backprojection procedure are exposed and supported with numerical simulations showing feasibility with real data. Results are already very encouraging considering the problem of nondestructive and noninvasive 3-D imaging of samples supported by a deep or dense material. Clearly, the current method should still be worked on to eliminate some artifacts which are strong enough to blur out some of the contrast of the electron density. Yet our methodology enables us to strengthen our detailed understanding of the image formation process and how it is 


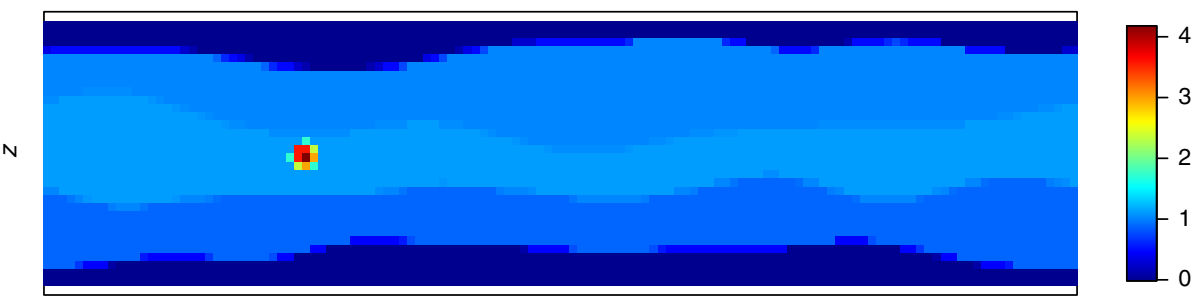

$x$
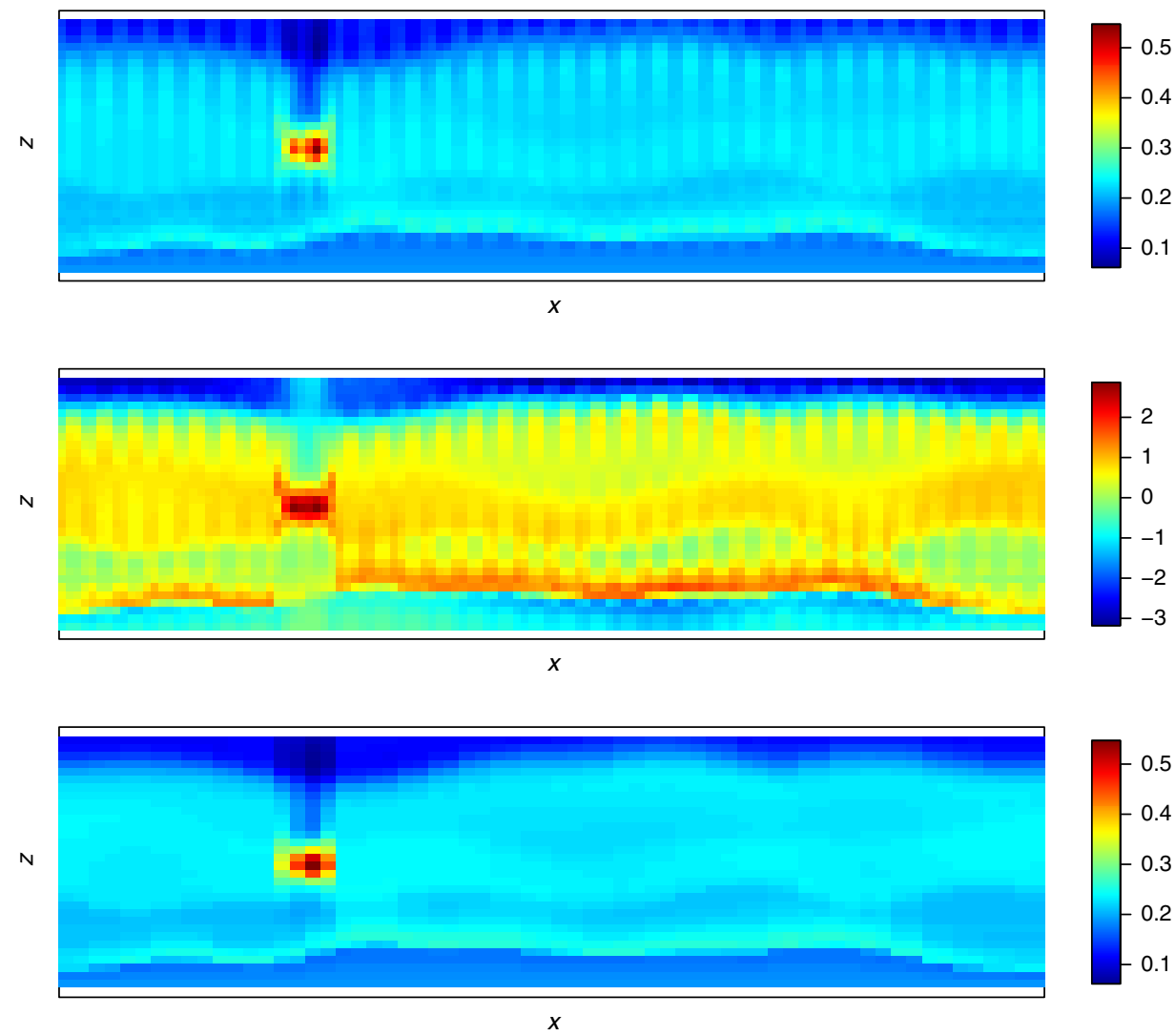

Fig. 8 Stratigraphical phantom of dimensions $128 \times 128 \times 32$ voxels. Plane presented: $y=25$. Same layout as in Fig. 7.

coupled to the volume reconstruction itself. This is proving instrumental in designing the physical instrument that will ultimately enable such imaging modality.

The current method assumes an exact relationship between the diffusion energy $E_{\omega}$ and the scattering angle $\omega$ according to Eq. (1). Through the Doppler effect and electron binding energy variations this relationship is indeed a non-Dirac probabilistic law. We will use a combination of Monte-Carlo simulations and variational analysis to assess the consequences of our approximation on image formation and volume reconstruction in a current work.

To be able to tackle the system computationally, we have first focused on flat objects and using incident energy that optimize the relative importance of single event inelastic scattering versus more complex multiple scatterings and absorption interactions. At the chosen incident energy, the probability of multiple scattering is very small since the mean free path of the x-ray photons is in the same order of magnitude as the probed volume thickness. When dealing with single scattering and a monochromatic incident beam, it is easy to discriminate photons generated by elastic versus inelastic scattering events. At the selected incident energy, the mean free path attached to scattering is much shorter than that attached to the photoelectric absorption process, i.e., the effect of photoelectric absorption is much smaller than that of scattering effects. It is important to note that inelastically scattered photons have an energy which is in the same domain as the incident photon energy when it comes to the relative importance of absorption and scattering.

Still we plan to work on an iterative reconstruction process which, while more expensive computationally, will enable us to also account for the smaller effects, photoelectric absorption and multiple scattering, in the produced reconstruction.

\section{Acknowledgments}

The authors would like to thank Pierre Gueriau and Mathieu Thoury for their support, discussions, and help to acquire images of heritage objects. Annelies van Loon for providing 
the image of the Rembrandt painting, and the field workers who collected the fossil actinopterygian. Patricio Guerrero would like to thank the French Fondation des Sciences $d u$ Patrimoine (PATRIMA LabEx) for providing a PhD grant to support his research work. The same author would also like to thank Javier Cebeiro for his crucial help and discussions.

\section{References}

1. P. Gueriau et al., "Trace elemental imaging of rare earth elements discriminates tissues at microscale in flat fossils," PLoS One 9(1), e86946 (2014)

2. M. K. Nguyen, T. T. Truong, and P. Grangeat, "Radon transforms on a class of cones with fixed axis direction," J. Phys. A: Math. Gen. 38(37), 8003-8015 (2005).

3. M. Haltmeier, "Exact reconstruction formulas for a radon transform over cones," Inverse Probl. 30(3), 035001 (2014).

4. J. Cebeiro, M. Morvidone, and M. K. Nguyen, "Back-projection inversion of a conical Radon transform," Inverse Probl. Sci. Eng. 24(2), 328$352(2015)$

5. S. J. Norton, “Compton scattering tomography," J. Appl. Phys. 76, $2007-$ 2015 (1994).

6. M. Morvidone et al., "On the V-line radon transform and its imaging applications," Int. J. Biomed. Imaging 2010, 11 (2010).

7. T. T. Truong and M. K. Nguyen, "On new V-line radon transforms in $\mathbb{R}^{2}$ and their inversion," J. Phys. A: Math. Theor. 44(7), 075206 (2011).

8. T. T. Truong and M. K. Nguyen, "New properties of the V-line radon transform and their imaging applications," J. Phys. A: Math. Theor. 48(40), 405204 (2015)

9. O. Klein and Y. Nishina, "The scattering of light by free electrons according to Dirac's new relativistic dynamics," Nature 122, 398-399 (1928).
Patricio Guerrero Prado has been a $\mathrm{PhD}$ candidate in computed tomography, Radon transforms, and applied mathematics since November 2014 at the European research platform on ancient materials IPANEMA under the direction of Serge Cohen, Mai Nguyen, and Laurent Dumas. His thesis title is "Compton 2D imaging for 3D reconstruction."

Mai K. Nguyen received her $\mathrm{PhD}$ in signal and image processing from Grenoble National Polytechnic Institute, France, in 1988. She has been a professor in the Department of Computer Sciences at the University of Cergy-Pontoise since 2005. Her research interests include inverse problems, generalized Radon transforms, and their applications in imaging science, scattered ionizing radiation imaging, biomedical imaging, nondestructive evaluation. She is an IEEE senior member.

Laurent Dumas received his $\mathrm{PhD}$ in applied mathematics from Paris Diderot University in 1995 . He has been a professor at Versailles University since 2010. His research interests include numerical analysis, derivative free optimization, uncertainty quantification, and have been applied in various engineering and medical fields.

Serge $\mathbf{X}$. Cohen earned his PhD in biophysics at EMBL Grenoble. After a postdoctorate period at NKI (Amsterdam) working on shape recognition and statistical learning applied to structural biology, he is now a mathematician at CNRS. Since 2010, leading information extraction and analysis scientists at IPANEMA, he has focused on theoretical statistics aspects for spectromicroscopy and synchrotron radiation tomography for ancient materials. 